\title{
Cognitive functioning is impaired in patients with chronic fatigue syndrome devoid of psychiatric disease
}

\author{
John DeLuca, Susan K Johnson, Steven P Ellis, Benjamin H Natelson
}

\begin{abstract}
Objective-To examine the effect of the presence or absence of psychiatric disease on cognitive functioning in chronic fatigue syndrome.

Methods-Thirty six patients with chronic fatigue syndrome and 31 healthy controls who did not exercise regularly were studied. Subgroups within the chronic fatigue syndrome sample were formed based on the presence or absence of comorbid axis I psychiatric disorders. Patients with psychiatric disorders preceding the onset chronic fatigue syndrome were excluded. Subjects were administered a battery of standardised neuropsychological tests as well as a structured psychiatric interview.

Results-Patients with chronic fatigue syndrome without psychiatric comorbidity were impaired relative to controls and patients with chronic fatigue syndrome with concurrent psychiatric disease on tests of memory, attention, and information processing.

Conclusion-Impaired cognition in chronic fatigue syndrome cannot be explained solely by the presence of a psychiatric condition.
\end{abstract}

(F Neurol Neurosurg Psychiatry 1997;62:151-155)

Keywords: chronic fatigue syndrome; cognition; psychiatric illness

Chronic fatigue syndrome is a disease which is characterised by persistent and debilitating fatigue, as well as neuropsychiatric, infectious, and rheumatological symptoms. No single pathogenic mechanism has been consistently identified using physical or laboratory tests, thus making the diagnosis of chronic fatigue syndrome one of exclusion. Although a case definition has been established by the Centers for Disease Control (CDC), ${ }^{12}$ the heterogeneity of the case defined chronic fatigue syndrome population may be one major contributing factor accounting for the lack of consistent medical findings. Thus seeking critical variables which may identify more homogeneous subgroups may not only increase the diagnostic accuracy in chronic fatigue syndrome but may help in the identification of potential causative agents. ${ }^{2}$ One such critical variable is psychiatric disease. Some studies have reported a relatively high frequency of psychiatric disorders (primarily depression) in those with chronic fatigue (not necessarily chronic fatigue syndrome). ${ }^{3-6}$ This, coupled with the fact that many symptoms of chronic fatigue syndrome resemble those of depression, has led to the notion that chronic fatigue syndrome is a primary psychiatric disorder. The purpose of the present study was to examine the effect of the presence or absence of psychiatric disease on cognitive functioning in chronic fatigue syndrome.

Cognitive difficulties can be the most disabling and troublesome aspects of chronic fatigue syndrome, ${ }^{5}$ with such complaints reported in up to $85 \%$ of patients. ${ }^{6}$ Several groups have reported cognitive impairment on objective neuropsychological testing. ${ }^{7-13}$ When detected, these impairments are generally subtle, and primarily in the area of attention and concentration, memory, and information processing efficiency. Because of the high rate of psychiatric disorders in patients with chronic fatigue syndrome, some research groups have indicated the need to clarify the role of depression in producing cognitive impairment. ${ }^{513-16}$ A similar recommendation was made by an international NIH/CDC study group, which further recommended the use of stratification techniques to consider this issue. ${ }^{2}$

To study the influence of psychiatric disease on cognitive functions in chronic fatigue syndrome, we examined two groups of patients: (1) those without psychiatric disorder(s) in their lifetime or concurrent with chronic fatigue syndrome: (CFS-nopsych group); and (2) those with a concurrent axis 1 psychiatric disorder, (CFS-psych group). If psychiatric disease is the primary reason for impaired cognitive functioning in chronic fatigue syndrome, the CFS-psych group should be more impaired relative to controls on neuropsychological testing than the CFS-nopsych group. By contrast, greater impairment in the CFSnopsych group than the CFS-psych group relative to controls would lend support to the claim by some that impaired cognition is due to cerebral dysfunction. ${ }^{11-13}$

\section{Methods}

SUBJECTS

We studied 36 patients with chronic fatigue syndrome and 31 healthy subjects who did not exercise regularly (controls). The groups did not differ statistically in mean age, sex distribution, or years of education (table 1). Patients with chronic fatigue syndrome were recruited via self referral based on media reports about the centre and by physician referral. Inclusion 
Table 1 Mean (SD) demographic and clinical characteristics

\begin{tabular}{lllllll}
\hline & $\begin{array}{l}\text { No of } \\
\text { patients }\end{array}$ & Age $(y)$ & $\begin{array}{l}\text { Sex } \\
(\% \text { female })\end{array}$ & Education $(y)$ & Fatigue & BDI \\
\hline CFS & 36 & $33 \cdot 6(8 \cdot 7)$ & $86 \%$ & $14 \cdot 7(2 \cdot 5)$ & $57 \cdot 7(4 \cdot 6)^{\star}$ & $14 \cdot 0(7 \cdot 7)^{\star}$ \\
CFS-psych & 15 & $31 \cdot 9(9 \cdot 5)$ & $86 \%$ & $14 \cdot 8(2 \cdot 3)$ & $58 \cdot 5(4 \cdot 2)^{\star}$ & $18 \cdot 6(8 \cdot 1)^{\star} \dagger$ \\
CFS-nopsych & 21 & $34 \cdot 8(8 \cdot 1)$ & $87 \%$ & $14 \cdot 7(2 \cdot 7)$ & $57 \cdot 2(4 \cdot 8)^{\star}$ & $10 \cdot 8(5 \cdot 6)^{\star}$ \\
Controls & 31 & $37 \cdot 3(10 \cdot 2)$ & $90 \%$ & $15 \cdot 8(2 \cdot 6)$ & $18 \cdot 9(10 \cdot 8)$ & $1 \cdot 8(2 \cdot 4)$ \\
\hline
\end{tabular}

*Significantly different from controls.

†Significantly different from CFS-nopsych group.

\Sample size as follows: CFS-psych $=12$, CFS-nopsych $=21$, Controls $=28$.

$\mathrm{BDI}=$ Beck depression inventory.

of patients with chronic fatigue syndrome was based on a careful history, physical examination, elimination of possible medical causes of fatigue, and fulfillment of the published case definition for chronic fatigue syndrome. ${ }^{117}$

Additional exclusion criteria were: (1) illness onset longer than four years; (2) symptoms of less than moderate severity at the time of intake; and (3) a history of a psychiatric disorder in the five years before the onset of chronic fatigue syndrome. This last criterion effectively eliminated all patients with chronic fatigue syndrome with prior psychiatric histories with the exception of one patient in the CFS-psych group who had had a major depressive episode many years earlier. Based on our experience, we exclude about $10 \%$ of our patients with chronic fatigue syndrome at intake (psychiatric interview) due to an axis I disorder in the five years before diagnosis. Healthy subjects were recruited by advertising in the local community, and were paid for their participation. Only those who reported no medical problems, no psychiatric history, and were not taking medication other than birth control pills were included as subjects.

Psychiatric history (DSM III-R axis I disorders) was obtained in all subjects using a structured psychiatric interview, the computerised version of the diagnostic interview schedule (DIS) ${ }^{18}$ administered by a psychologist or neuropsychology technician trained in its use. Additional exclusions included a history of loss of consciousness for greater than five minutes, substance misuse, eating disorders, schizophrenia, or bipolar disorder.

\section{PROCEDURE}

The patients with chronic fatigue syndrome were divided into those who had a DSM III-R axis I psychiatric diagnosis occurring since their diagnosis (but not in the five years before diagnosis) (CFS-psych: $\mathbf{n}=15$ ) and those without a psychiatric diagnosis, either concurrently or historically; CFS-nopsych: $n=21$ ). The primary axis I diagnosis was major depression, found in $73 \%$ of the CFS-psych group. Other axis I disorders included dysthymia (13\%), phobia (26\%), panic disorder $(26 \%)$, generalised anxiety disorder $(20 \%)$, and somatoform disorder (6\%) (percentages do not add up to $100 \%$ because patients may have had more than one diagnosis). There were no significant differences between the two chronic fatigue syndrome subgroups with respect to age, sex distribution, and education. Mean level of depression (Beck depression inventory) was significantly raised in the two chronic fatigue syndrome groups relative to controls $(F(2,64)=56 \cdot 81, \quad \mathrm{P}=0.0001)$. Mean level of depression was also significantly higher in the CFS-psych group than in the CSF-nopsych group $(P=0.0035)$. As expected, fatigue was significantly greater in the two chronic fatigue syndrome groups than in controls $(F(2,57)=168 \cdot 8, \mathrm{P}=0 \cdot 0001)$, but the CSF-psych group and the CSFnopsych group did not differ from each other (table 1).

All subjects were given a battery of standard neuropsychological tests, administered and scored in accordance with published procedures. The tests consisted of the paced auditory serial addition test (PASAT), assessing complex information processing efficiency; vocabulary, arithmetic, and digit span subtests of the WAIS-R, ${ }^{19}$ assessing intellectual and attentional skills; the Rey-Osterreith complex figure test (ROCFT), assessing visual memory and visual-constructional ability; and the California verbal learning test (CVLT), ${ }^{20}$ measuring verbal memory. The Beck depression inventory $(\mathrm{BDI})^{21}$ and the fatigue severity scale $^{22}$ were also administered.

\section{DATA ANALYSIS}

The 12 dependent variables of the neuropsychological data were analysed by multiple analysis of covariance (MANCOVA) with subject condition (CFS-nopsych, CFS-psych, controls) as the between group factor, and age, sex, and education as covariates. If the subject condition factor on the MANCOVA was significant, subsequent analyses consisted of separate one way analyses of covariance (ANCOVAs) for each neuropsychological variable, with group (CFS-psych, CFSnopsych, and controls) as the between group factor and age, education, and sex as covariates. Post-hoc analyses were conducted using the Welch modified $t$ test. $^{23}$

\section{Results}

The overall MANCOVA showed that the factor of subject condition was significantly different for overall neuropsychological performance $\quad(F \quad(24,102)=2 \cdot 13, \quad \mathrm{P}=$ $0.005)$. Post hoc MANCOVAs showed that both the CFS-nopsych $(P=0.003)$ and CFSpsych groups $(P=0.049)$ differed from the control group, but not from each other.

To examine which of the specific tests were influenced across the three groups, the 
Table 2 Mean (SEM) neuropsychological performance for the CFS-nopsych group, CFS-psych group, and controls

\begin{tabular}{|c|c|c|c|c|c|}
\hline & $\begin{array}{l}\text { CFS-nopsych } \\
(n=21)\end{array}$ & $\begin{array}{l}C F S-p s y c h \\
(n=15)\end{array}$ & $\begin{array}{l}\text { Controls } \\
(n=31)\end{array}$ & $F$ value & $P$ value \\
\hline \multicolumn{6}{|l|}{ Rey figure: } \\
\hline Copy & $67 \cdot 4(1 \cdot 2)$ & $68 \cdot 2(1 \cdot 1)$ & $68.9(0.5)$ & 0.89 & NS \\
\hline Immediate recall & $33 \cdot 2(2 \cdot 8) t^{\star}$ & $42 \cdot 3(3 \cdot 0)$ & $44 \cdot 8(1 \cdot 6)$ & $7 \cdot 68$ & 0.001 \\
\hline Delayed recall & $32.5(2.9) \ddagger^{\star}$ & $43 \cdot 3(2 \cdot 8)$ & $41 \cdot 4(2 \cdot 0)$ & $4 \cdot 90$ & 0.01 \\
\hline \multicolumn{6}{|l|}{ CVLT: } \\
\hline T score & $32.3(3.6)^{\star}$ & $41 \cdot 4(3 \cdot 8)$ & $48 \cdot 2(1 \cdot 8)$ & $8 \cdot 70$ & 0.0005 \\
\hline Short free recall & $9.3(0.8)^{\star} \dagger$ & $11.7(0.7)$ & $12.3(0.5)$ & 6.49 & 0.0028 \\
\hline Long free recall & $10.2(0.8)^{\star}$ & $11.7(0.7)$ & $12.9(0.5)$ & $5 \cdot 12$ & 0.009 \\
\hline PASAT (total) & $125 \cdot 8(8 \cdot 2)^{\star}$ & $135 \cdot 4(4 \cdot 7)$ & $147.4(4.0)$ & $4 \cdot 74$ & 0.012 \\
\hline \multicolumn{6}{|l|}{ Digit span: } \\
\hline Forward & $9 \cdot 0(0 \cdot 6)$ & $9.9(0.8)$ & $9.9(0 \cdot 3)$ & 1.51 & NS \\
\hline Backward & $7.0(0.5)^{\star} \dagger$ & $9 \cdot 1(0 \cdot 7)$ & $8.8(0.4)$ & $4 \cdot 82$ & 0.011 \\
\hline Arithmetic (ss) & $12 \cdot 4(0 \cdot 8)$ & $14.3(0 \cdot 8)$ & $13.5(0.6)$ & $2 \cdot 00$ & NS \\
\hline Block design & $32 \cdot 6(1 \cdot 6)$ & $35 \cdot 5(2 \cdot 1)$ & $33 \cdot 2(1 \cdot 3)$ & $0 \cdot 74$ & NS \\
\hline Vocabulary & $51 \cdot 1(2 \cdot 1)$ & $54 \cdot 5(2 \cdot 2)$ & $53.5(1.5)$ & 0.39 & NS \\
\hline
\end{tabular}

$\star P \leqslant 0.01 v$ controls.

$\dagger$
$\pm \mathrm{P} \leqslant 0.05 v$ CFS-psych.

Age, sex, and education were included as covariates in the statistical analysis, ss = standard score (age corrected). drome is influenced by the presence or absence of an axis I psychiatric diagnosis (primarily major depression). The results clearly showed the existence of important subgroups within a sample of patients who met the case definition for a diagnosis of chronic fatigue syndrome. The overall result showed that, relative to healthy controls, cognition was impaired in the CFS-nopsych group (chronic fatigue syndrome subjects without a lifetime or concurrent psychiatric disorder). Patients with chronic fatigue syndrome with a concurrent axis 1 psychiatric diagnosis (CFS-psych group) did not differ from controls on individual neuropsychological tests. Further, the CFS-nopsych group also performed significantly below the CFS-psych group on several measures of memory and concentration. These data suggest that cognitive impairment in chronic fatigue syndrome cannot simply be explained by the presence of psychiatric state, and are contrary to expectations based on a model of depression induced cognitive impairment in chronic fatigue syndrome.

It should be noted that whereas the CFSpsych group did not differ significantly from controls on individual tests of cognition, when the neuropsychological tests were analysed as a whole (by MANCOVA), a statistical difference did emerge. This suggests that although the CFS-psych group may show a small decrement in "overall" cognitive functioning, this effect is very subtle and unlikely to be seen in individual patients undergoing clinical testing.

The results of the present study are particularly important in the light of the recommendations by an international NIH/CDC study group, ${ }^{2}$ which outlined the need to clarify the role of comorbid psychiatric conditions in symptoms of chronic fatigue syndrome. Previous researchers have specifically emphasised the need to clarify the role of psychiatric disorders on cognitive functioning in chronic fatigue syndrome. ${ }^{513-15}$ The technique of using statistical measures to adjust for the presence of depression on cognitive performance has provided no clear solution; although several studies ${ }^{711-13}$ have found no relation between depression and cognitive performance, others $^{14} 16$ have. To our knowledge, the present study is the first to report significant differences in cognitive performance in patients with chronic fatigue syndrome with and without comorbid psychiatric disorders. The only other study comparing patients with chronic fatigue syndrome with and without "significant depression" did not find impaired neuropsychological performance in either group compared with healthy and depressed controls. ${ }^{24}$ However, there are some important differences between the two studies which may account for the discrepant findings. The study of Cope et $a l^{24}$ (1) did not exclude subjects with concurrent psychiatric disorders (other than "significant depression") in their chronic fatigue group without depression; (2) did not exclude subjects with prior psychiatric disorders; and (3) did not require patients to meet the more stringent CDC case definition ${ }^{117}$ for chronic fatigue syndrome. 
At first glance, the finding that patients with chronic fatigue syndrome with comorbid psychiatric disturbance were not impaired on tests of cognition seems counterintuitive. This is because of the common belief that psychopathology (primarily depression) itself results in cognitive impairment. However, little convincing evidence to support this belief exists with some ${ }^{25-27}$ but not all studies ${ }^{24}$ 28-30 showing impaired cognitive functioning in depressed patients on effortful tasks. On the contrary, studies in several medical populations $^{31-34}$ and even among currently healthy subjects (with personal or family psychiatric history $)^{35}$ show no adverse effects of depression on cognitive functioning.

The results of the present study have important implications for treatment. That is, patients with chronic fatigue syndrome with psychiatric complications may benefit more from psychotherapy in conjunction with psychopharmacological interventions. By contrast, patients without psychiatric comorbidity may benefit more from a psychoeducational approach to symptom management or cognitive rehabilitation.

The present results can be added to a growing body of literature suggesting that at least some patients with chronic fatigue syndrome differ from patients with major depression. ${ }^{36-41}$ Taken together, the results of the present study suggest that at least in a subgroup of patients, chronic fatigue syndrome is not simply a manifestation of a primary psychiatric disorder such as major depression. Although the results of the present study do not provide direct evidence for an encephalitic process as suggested by others, ${ }^{42-45}$ our working hypothesis is that patients with chronic fatigue syndrome without concurrent or history of psychopathology is the subgroup with the highest probability of finding reproducible biomedical markers.

In the present study, stratification of patients with chronic fatigue syndrome by the presence of comorbid psychiatric disorder clearly reduced the heterogeneity of the chronic fatigue syndrome population, which had important consequences on measures of cognitive functioning. Employing a stratification methodology contrasts with another recently suggested approach to studying chronic fatigue syndrome. Katon and Russo ${ }^{46}$ noted that patients with chronic fatigue syndrome reporting many symptoms also had a high lifetime prevalence of psychiatric disorders. Their approach to considering this problem was to redefine chronic fatigue syndrome by reducing the number of required symptoms. However, the net effect of doing this would be to increase population heterogeneity. The present data argue for accepting patients with many symptoms but stratifying the sample for lifetime and concurrent psychiatric diagnoses.

We thank Ms Dawn Beldowicz for help in data collection. This tudy was supported in part by grants AI-32247 and H133P10002-92 from the National Institutes of Health and rant H129J10006 from the Rehabilitation Services Administration.
1 Holmes GP, Kaplan JR, Gantz NM, et al. Chronic fatigue syndrome: a working case definition. Ann Intern Med 1988;108:387-9.

2 Fukuda K, Staus SE, Hickie I, Sharpe MC, Dobbins JG, Komaroff $A$. The chronic fatigue syndrome: a comprehensive approach to its definition and study. Ann Intern Med 1994;121:953-9.

3 Manu P, Matthews DA, Lane TJ. The mental health of patients with a chief complaint of chronic fatigue: a prospective evaluation and follow-up. Arch Intern Med 1988;148:2213-7.

4 Manu P, Lane TJ, Matthews DA. Chronic fatigue syndromes in clinical practice. Psychother Psychosom 1992; 58:60-8.

5 Abbey SE, Garfinkle PE. Chronic fatigue syndrome and depression: cause, effect, or covariate. Rev Infect Dis 1991;13(suppl 1):S73-83.

6 Grafman J. Neuropsychological features of chronic fatigue syndrome. In: Straus SE, ed. Chronic fatigue syndrome. syndrome. In: Straus SE, ed. Chronic fati

7 DeLuca J, Johnson SK, Natelson $\mathrm{BH}$. Information processing in chronic fatigue syndrome and multiple sclerosis. Arch Neurol 1993;50:301-4.

8 DeLuca J, Johnson SK, Beldowicz D, Natelson BH. Neuropsychological impairments in chronic fatigue syndrome, multiple sclerosis, and depression. If Neurol Neurosurg Psychiatry 1995;58:38-43.

9 Ray C, Phillips L, Weir WRC. Quality of attention in chronic fatigue syndrome: subjective reports of everyday attention and cognitive difficulty, and performance on
tasks of focused attention. Br $\mathcal{F}$ Clin Psychol 1993;32: 357-64.

10 Grafman J, Schwartz V, Dale JK, Scheffers M, Houser C, Straus SE. Analysis of neuropsychological functioning in patients with chronic fatigue syndrome. $₹$ Neurol patients with chronic fatigue sy
Neurosurg Psychiatry 1993;56:684-9.

11 Sandman CA, Barton JL, Nackoul K, Goldstein J, Fidler F. Memory deficits associated with chronic fatigue immune dysfunction syndrome. Biol Psychiatry 1993;33:618-23.

12 Smith AP, Behan PO, Bell W, Millar K, Bakheit M Behavioral problems associated with the chronic fatigue syndrome. Br F Psychol 1993;84;41 1-23.

13 Riccio M, Thompson C, Wilson B, Morgan DJ, Larient AF. Neuropsychological and psychiatric abnormalities in myalgic encephalomyelitis: a preliminary report. $\mathrm{Br} f \mathrm{Clin}$ Psychol 1992;31:111-20.

14 McDonald E, Cope H, David A. Cognitive impairment in patients with chronic fatigue: a preliminary study. $f$ Neurol Neurosurg Psychiatry 1993;56:812-5.

15 Grafman J, Johnson R, Scheffers $M$. Cognitive and moodstate changes in patients with chronic fatigue syndrome. Rev Infect Dis 1991;13(suppl 1):S45-52.

16 Krupp LBB, Sliwinski M, Masur DM, Friedberg F, Coyle PK. Cognitive functioning and depression in patients $\mathrm{PK}$. Cognitive functioning and depression in patients
with chronic fatigue syndrome and multiple sclerosis. with chronic fatigue syndrome

17 Schluederberg A, Straus SE, Peterson P, et al. Chronic fatigue syndrome: definition and medical outcome assessment. Ann Intern Med 1992;117:325-31.

18 Marcus, S, Robins, LN, Bucholz, K. Quick diagnostic interview schedule III-R, version 1. St Louis, MO: Washington University School of Medicine, 1990.

19 Wechsler D. Wechsler adult intelligence scale-revised, manual. New York, Psychological Corporation, 1981.

20 Lezak M. Neuropsychological assessment. New York: Oxford University Press, 1995.

21 Beck AT, Ward CH, Mendelson M, Mock J, Erbaugh J. An inventory for measuring depression. Arch Gen Psychiatry 1961;4:561-71.

22 Krupp LB, LaRocca NG, Muir-Nash J, Steinberg AD. The fatigue severity scale: application to patients with multiple sclerosis, and systemic lupus erythematosus. Arch ple sclerosis, and systemi

23 Rice JA. Mathematical statistics and data analysis. Pacific Grove, CA: Wadsworth and Brooks/Cole, 1988.

24 Cope H, Pernet A, Kendall B, Davis A. Cognitive functioning and magnetic resonance imaging in chronic fatigue syndrome. Br $\mathcal{F}$ Psychiatry 1995;167:86-94

25 Cohen RM, Weingartner H, Smallberg SA, Pickar D, Murphy D. Effort and cognition in depression. Arch Gen Psychiatry 1982;144:376-82.

26 Golinkoff M, Sweeney JA. Cognitive impairments in depression. $\mathcal{F}$ Affect Disord 1989;17:105-12

27 Tancer ME, Brown TM, Evans DL, Eckstrom D, Haggerty JJ Jr, Pederson C, et al. Impaired effortful cognition in depression. Psychiatry Res 1990;31:161-8.

28 Grossman I, Kaufman AS, Mednistky S, Scharff L, Dennis B. Neurocognitive abilities for a clinically depressed sample versus a matched control group of normal individuals. Psychiatric Res 1994;51:231-44.

29 Kaufman AS, Grossman I, Kaufman NL. Comparison of hospitalized depressed patients and matched normal con-
trols on tests that differ in their level of cognitive comtrols on tests that differ in their level of cognitive com-
plexity. fournal of Psychoeducational Assessment 1994;12: plexity.

30 Miller LS, Faustman WO, Moses JA, Csernansky JG. Evaluating cognitive impairment in depression with the Evaluating cognitive impairment in depression with the
Luria-Nebraska neuropsychological battery: severity corLuria-Nebraska neuropsychological battery: severity correlates and comparisons with

31 Grafman J, Rao SM, Litvan I. Disorders of memory. In: Rao SM, ed. Neurobehavioral aspects of multiple sclerosis. New York: Oxford University Press, 1990: 102-17. 
32 DeLuca J, Johnson SK. Cognitive impairments in multiple sclerosis: implications for rehabilitation Neurorehabilitation 1993;3:9-16.

33 Davidoff GN, Roth EJ, Richards JS. Cognitive deficits in spinal cord injury: epidemiology and outcome. Arch Phys Med Rehabil 1992;73:275-84.

34 Bornstein RA, Pace P, Rosenberger P, Nasrallah HA, Para MF, Whitacre CC, Fass RJ. Depression and neuropsyMF, Whitacre CC, Fass RJ. Depression and neuropsy-
chological performance in asymptomatic HIV infection Am ₹ Psychiatry 1993;150:922-7.

35 Purcell DW, Schwartz AJ, Brookshire RJ, Cardler J Lewine RRJ. Neuropsychological functioning in "normal" subjects. Neuropsychiatry, Neuropsychology, and Behavioural Neurology 1995;8:6-13.

36 Natelson BH, DeLuca J, Johnson SK, et al. Reducing heterogeneity in the chronic fatigue syndrome: a comparison with deprerssion and multiple sclerosis. Clin Infect Dis 1995;21:1204-10.

37 Johnson SK, DeLuca J, Natelson BH. Personality dimensions in the chronic fatigue syndrome: a comparison with multiple sclerosis. F Psychiatr Res 1996;30:9-20.

38 Johnson SK, DeLuca J, Natelson BH. Depression in fatiguing illness: comparing patients with chronic fatigue syndrome, multiple sclerosis and depression. $\mathcal{F}$ Affect Disord 1996;39:21-30.

39 Pepper CM, Krupp LB, Friedberg F, Doscher C, Coyle PK. A comparison of neuropsychiatric characteristics in chronic fatigue syndrome, multiple sclerosis, and major depression. F Neuropsychiatry Clin Neurosci 1993;5:200-5. 40 Powell R, Dolan R, Wessely S. Attributions and self-esteem in depression and chronic fatigue syndrome. $\mathcal{F}$ Psychosom Res 1990;21:665-73.

41 Cleare AJ, Bearn J, McGregor A, Allain T, Wessely S, Murray RM, et al. Contrasting neuroendocrine responses in depression and chronic fatigue syndrome. $\mathcal{f}$ Affect in depression and chron

42 Natelson BH, Cohen JM, Brassloff I, Lee HJ. A controlled study of brain magnetic resonance imaging in patients with fatiguing illnesses. F Neurol Sci 1993;120:213-7.

43 Buchwald DPR, Cheney DI, Peterson B, et al. A chronic illness characterized by fatigue, neurologic and immunologic disorders and active herpes virus type 6 infection. Ann Intern Med 1992;116:103-13.

44 Ichese M, Salit IE, Abbey SE, et al. Assessment of regional cerebral perfusion by ${ }^{99} \mathrm{Tc}^{\mathrm{m}}$-HMPAO SPECT in chronic fatigue syndrome. Nucl Med Commun 1992;13:767-72.

45 Schwartz RB, Garada BM, Komaroff AL, Tice HM, Gleit $\mathrm{M}$, Jolesz FA, et al. Detection of intracranial abnormalities in patients with chronic fatigue syndrome: comparison of MRI imaging and SPECT. American Roentgen Ray Society 1994;162:935-41.

46 Katon W, Russo J. Chronic fatigue syndrome criteria: a critique of the requirement for multiple physical complaints. Arch Intern Med 1992;152:1604-9. 